\title{
Research on the Application of Machine Learning Algorithm and Fuzzy Logic in Eating Assistive Robot
}

\author{
Mubashar Nawaz, Xianhua Li, Sohaib Latif, Sadaf Irshad, Shabnam Sarwar
}

\begin{abstract}
More than 110 million people in this world are facing some kind of disability, for which they experience difficulty while eating food. Eating Assistive Robots could meet the needs of the elderly and people with upper limb disabilities or dysfunctions in gaining independence in eating. We are researching making a robot, which can assist the disabled in eating their meals. Our Eating Assistive Robot will detect the face of the disabled and process it for whether his/her mouth is opened or closed. Our robot will put a pre-prepared replaceable spoon of food in his/her mouth iteratively until the food lasts in the food container. The methodology we used for it i.e. firstly there is a live camera feed through which we are detecting human faces, after this, a library of Affectiva calculates how much mouth is open. We have set a certain threshold after which the program starts the stepper motor which brings the pre-filled spoon of food into the mouth of the disabled.
\end{abstract}

Keywords: Eating Assistive Robot, Fuzzification, Assistive Feeding, Machine Learning

\section{INTRODUCTION}

Eating Assistive Robots should meet the needs of the elderly and people with upper limb disabilities or dysfunctions in gaining independence in eating. We have researched how robots can assist the disabled conveniently in eating. We also have researched Robotics' Components and their integration with developed software. Eventually, we have developed an algorithm using an Affective library that detects a face and calculates how much percent the mouth is opened, and then we have built hardware that performs physical motions.

\subsection{Selective Attention}

It selects personal designated attention from multiple faces but does not stop or interrupt process information at the same time. It continuously processes the external environment camera feed for searching the human faces and to calculate the attention rate of each face.

Manuscript received on October 07, 2021.

Revised Manuscript received on October 11, 2021.

Manuscript published on October 30, 2021.

* Correspondence Author

Mubashar Nawaz, School of Mechanical Engineering, Anhui University of Science and Technology, Huainan, China

Xianhua Li, School of Mechanical Engineering, Anhui University of Science and Technology, Huainan, China

Sohaib Latif*, School of Mathematics and Big Data, Anhui University of Science and Technology, Huainan, China

Sadaf Irshad, School of Mathematics and Big Data, Anhui University of Science and Technology, Huainan, China

Shabnam Sarwar, School of Mathematics and Big Data, Anhui University of Science and Technology, Huainan, China

(c) The Authors. Published by Blue Eyes Intelligence Engineering and Sciences Publication (BEIESP). This is an open access article under the CC BY-NC-ND license (http://creativecommons.org/licenses/by-nc-nd/4.0/)
Then decide to whom it should give attention and to whom it should not attend. Attention selectivity and its relevance to goal-oriented behavior have become the most explored areas in western psychology and neuroscience research.

\subsubsection{Principle}

Motivated by the attention mechanism of humans i.e. how humans pay attention to a specific human, in interaction with multiple humans. We explored various psychological theories, related research papers, and algorithms to understand the human emotional attention mechanism. Then using the Viola-Jones algorithm face detection algorithm, we coded and integrated various units. We can demonstrate that the ac-claimed algorithm working on a video as input for the detection of emotional attention is possible. The results may or may not hold perfect in reality. But still, these results are reliable enough to use for our purpose.

\subsection{Attention Dependency Factors Problem}

There are a lot of factors on which attention could depend and divert from one to another person like motion, sound, facial expressions, and emotions. Humans normally consider all these factors to give attention e.g. a sudden voice could divert one's attention towards the source of voice or hand motion of a person to other could get his attention.

\subsubsection{Solution}

"Face is the transmitter of body and eyes are a transmitter of the face."

But in the case of interaction between multiple persons and a robot in specific environment human facial expressions plays an important role in attention diversion from one person to another. This allows us to focus upon face emotions and expressions as reflects the complete human body feelings and interests. Face provides enough information about the state of the individual.

\subsubsection{Weakness}

The weak point of this solution is that only facial emotions and expressions are being used to detect the attention of an individual. Attention is a vast term and neglecting other attention factors is not less than unfair with an individual. Thus, sound, linguistic, and motion can also be taken as input in future work.

\subsection{Face Detection Problem}

The Viola-Jones algorithm is not perfect. Its perfection is 90 percent for front faces. Sometimes, faces are detected in walls, chairs, shirts, and other likewise.

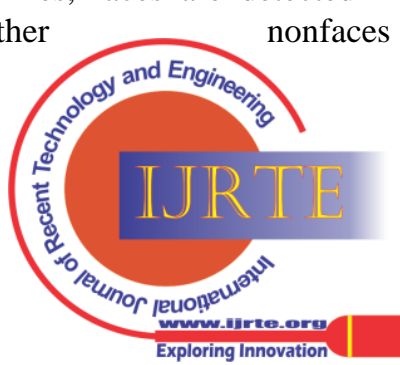




\subsubsection{Solution}

First, detect the face Detect Mouth/Lips if the mouth is detected. It's real face else it's a non-human face. Eyes can also be detected to make sure that it's a human face or not. This basic filtering is applied to make things workable and easy.

\subsection{Effects Of Changes In Head Angles And Face Luminance}

The best performance is in a range of +/- 25 degrees frontal, outside of which accuracy begins to suffer. Furthermore, for the effect of the differences in lighting conditions on the accuracy, the classifier accuracy drops only when the face is extremely poorly illuminated or backlit. This occurs when the average pixel brightness of the face falls below 30 on a scale of 0 (pitch black) to 255 (white).

\subsection{Cultural Differences}

Many scientific studies demonstrate the universality of facial expressions of emotions; however, each culture employs what we call "display rules" - culturally-specific rules that govern when people amplify, dampen or altogether mask a facial expression of emotion. The research demonstrating the effect of display rules is extensive, covers the past 50 years, and is widely acknowledged. In Southeast Asia there are very clear display rules around how to display emotion, especially in the presence of strangers (a work meeting, a moderator in a research study, etc.): namely, dampen their expressions, especially negative ones.

\subsubsection{Solution}

The algorithm is trained against a massive emotion data repository consisting of millions of faces that reflect data from 75 countries. This has hardened technology to account for cultural differences with high accuracy.

\section{LITERATURE REVIEW}

The therapy method is used to provide the robot with a rehabilitation and hand-off process for monitoring, guiding, and helping the post-stroke patients in order of rehabilitation process. This study provides us the opportunity for richer learning and adaptation algorithm[1].The robot navigates continuously the robot's arm activity and helps the patients remembering to copy the rehabilitation program. The second pilot study also described that there is a big relationship between physical embodiment and co-located robots [2].The novel system is designed to provide visually guided active feeding assistance and smoothly put food inside the user's mouth after being visually guided.The system is automatic for feeding patients by using motor impairments [3].A robot is consists of three distinguishing points: handling rice, a meal tray, and a modular design, which is consists of two arms. It can depend on the user's choice that which type of method he wants to chose [4].Brian 2.1 robot is a motivational robot which is used to helping in eating to older persons by its amazing voice and attractions. Many people like this experience and gave positive feedback [5].In $3 \mathrm{D}$ visualization, the virtual model is important to understanding the dynamic behavior and the position control of end effectors' position in the operation. The current system depends on the system requirements but can be changed in a virtual experimental robot [6].The system is based on a PR2 robot which provides a high-level interface by using the web interface to easily use it for people [7].By using this channel, there are three things in this system that are important in future work: User attraction, use of advanced technology, and selection of food items. In previous inquiries, there is the lake of technological work and limitations of selected food items[8].The user's facial expressions method is used to determine the user's demand and desire for those people who are paralyzed. The base-line algorithm is working properly in the face detection method which is currently using most the researchers in commercially available robots[9]. The amazing factor is the multiple uses of sensors, which are creating a smooth behavior with nature. This method is enhancing to create a wide functional behavior in a globally accepted systems [10].In our process; Assistive capabilities are included for tangible progress. This system work with motor impairments and is good for bodies users.PR2 robot is used with 2D cursor-based interfaces which provide accessibility for most users [11].The wince method is used for understanding the gestures of a user with a higher vision process. This method has a feasible approach as compare to older methods due to its high accuracy and speedy work [12].Due to an increase in pressure, wiggling has faster penetration. Bite timing is an important to factor which depends on chewing and talking with someone so, adjustment can be made according to user behavior[13].The chosen system depends on a BCI based on the O300 paradigm for the outclass order detection. Software consist of two mainly principle sensors: one is kindest for environment perception and the second is Kenova Jacob for lightweight robot manipulation [14].The developed system is fully friendly of feeding a user by using a spoon and we have chosen multiple food items like rice, peanuts with highly recommended feedback for ensuring that the spoon is full or not [15].We analyzed that people like profound motor deficits for personal use. We used a robot out of the laboratory to serve in the home to settle and serve different tasks with the use of a web browser[16].A multimodal execution monitor has been introduced to assisting a robot for patient feeding. Six baseline methods were used for serving the disabilities[17].For 3D face detection, the setup of the dry electrode EEG system used to determine the patient's brain and the robot was fast, reliable, and has the continuous capacity to deliver stable signals during all process of experiment. Dry-electrode is more efficient as compare to wet-electrode for a long time running and smooth working [18].Discriminative optimization (DO) is a method to solve vision problems by updating the new examples and technology.For the $3 \mathrm{D}$ vision factor, we evaluated the potential of DO to solve problems under the rigidity of point cloud registration and proved that it is outperformed state of art approaches [19].For understanding facial behavior, open face source is the best technique in computer vision. Graphical command lines are used to explore the face angle and convert it's indifferent small graphic points for understanding face expressions[20]. 
The strategy has two main procedures: sim to real and second is real to sim. The first method is used in simulation as real-world applications and the second method is updating the methods for connecting the simulation with the real world[21].The robot shows the modular way in fit with research areas properly with different customized methods[22].ROS-based systems have been used to an enabled an unprecedented level of freedom for enhancing the level of visualization and interaction in the new platform. JavaScript is more valuable programming in this ROS robot with the new standards [23].

\section{PROPOSED METHOD}

The complete procedure for calculating attention includes steps like face detection and feature detection robot. Detection of the face has been done through C\# code. After getting different types of values through the live camera feed, passed these values as input to Matlab fuzzifier. This fuzzifier returns attention numeric value after applying fuzzy logic rules, as output. Then all these values and attention as output are shown in a graph. The library used for detecting face, the promise of providing 34 facial points on a detected human face. The attention through facial expressions decided on the following basis.

\subsection{Detection}

\subsubsection{Face Detection}

Face detection is performed using the Viola-Jones face detection algorithm. Landmark detection is then applied to each facial bounding box and 34 landmarks are identified. If the confidence of the landmark detection is below a threshold then the bounding box is ignored. Once a face is detected a boundary box is obtained around it. The boundary bow of the detected face moves with the movement of the face. Detection of faces and localization of the key facial landmarks have been done for each face. Extraction of texture features using HOG. Classification of facial actions

\subsubsection{Open Mouth Calculation}

This coding was built on the EMFACS emotional facial action coding system. The calculations are given a similar score from 0 (absent) to 100 (present).

\subsubsection{Face Size}

This face detection algorithm has an additional feature i.e. it can detect both small and large faces.

\subsubsection{Multiple Face Detection}

Our application has support for detecting faces up to 20 and can extract emotions, to give attention, in case they have head orientation within range.

3.2 Head OrientationIt can detect the human face within a specific range of angles. If head orientation is such that it is out of its angle range then it could not able to detect face neither the emotions nor other features. It detects emotions and other facial features with accuracy if the face is within +/- 25 degree angle.

\subsection{Brightness}

It works in a certain amount of light and when light intensity is below a certain threshold then it is not able to detect faces within the camera frame.

\section{FUZZIFICATION}

The modern-day technologies in the areas of information storage and retrieval, web search, image processing, control, pattern recognition, bio information and computational biology, e-markets, autonomous navigation, and guidance are benefited using the fuzzy logic systems. Fuzzy Logic (FL) is a method of reasoning that resembles human reasoning. The approach of FL imitates the way of decisionmaking in humans that involves all intermediate possibilities between digital values YES and NO.

The process of fuzzification is of great importance for getting the required results. As described in the above section, calculation of attention has been done through fuzzy logic. We have used this in such a way that, two largest emotions numeric values (out of six emotions) of a human face, identified through a function used as input to the fuzzy logic system. For these two inputs, 25 rules have been written to get the required output as attention. Using these rules we get attention as output. Furthermore, dot net (.net) library has been made in Matlab, for further processing.

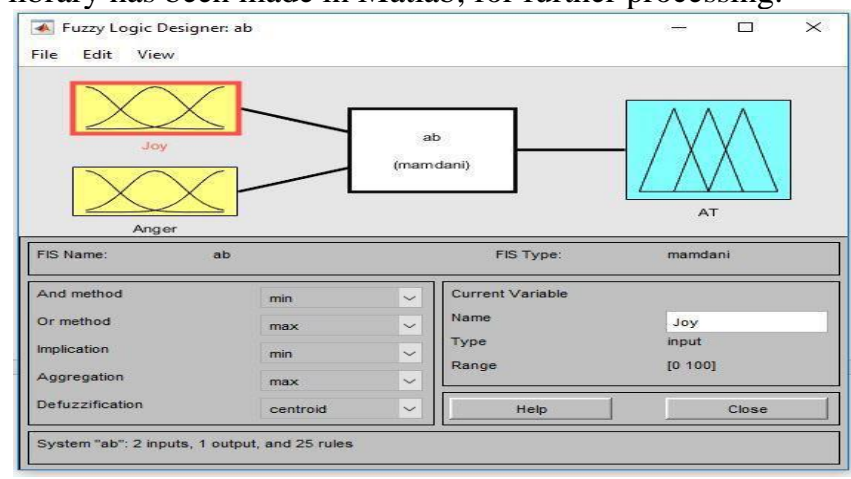

Figure 3.1: Fuzzy System for Attention

In figure 3.1 the abstract model of fuzzy inference system for calculating attention from the two largest emotions values of the detected human face has shown. One can see clearly from the figure that two emotion values are using here as input namely joy and anger and these values passed further. After applying Mamdani fuzzy rules to these inputs attention we get as output. Here in our scenario, the attention levels have been calculated through a fuzzy inference system, where the inputs, outputs, and there ranges for varying linguistic values are as under:

Joy=\{"Very Low", "Low", "Medium", "High", "Very High"\} $[0,1]$

Anger $=\{$ "Very Low", "Low", "Medium", "High", "Very High" $\}[0,1]$

Direct_Attention = $\{$ "Very Low", "Low", "Medium", "High", "Very High" $\}[0,1]$

3.2: Ranges for Fuzzy Inference System for calculating weights of direct attention In the above figure, 3.2 mentioned dimensions are arranged for all possible combinations to construct rules for Inference Engine. The possible rules fed to the fuzzy system are written in Matlab.

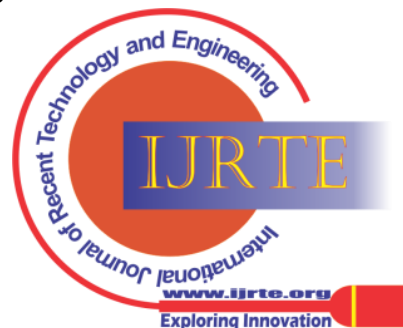




\subsection{Membership Function}

A membership function (MF) is a curve that defines in figure 3.3 how each point in the input space (also called the universe of discourse) is mapped to a membership value (or degree of membership) between 0 and 1 . $\mu A \cap I \cap B \cap S(x)=\min [\mu A(x), \mu I(x), \mu B(x), \mu S(x)]$

Figure 3.3: Mathematical \& Graphical MF of Layer-1 Emotional Attention-Fuzzy Inference System Input variables

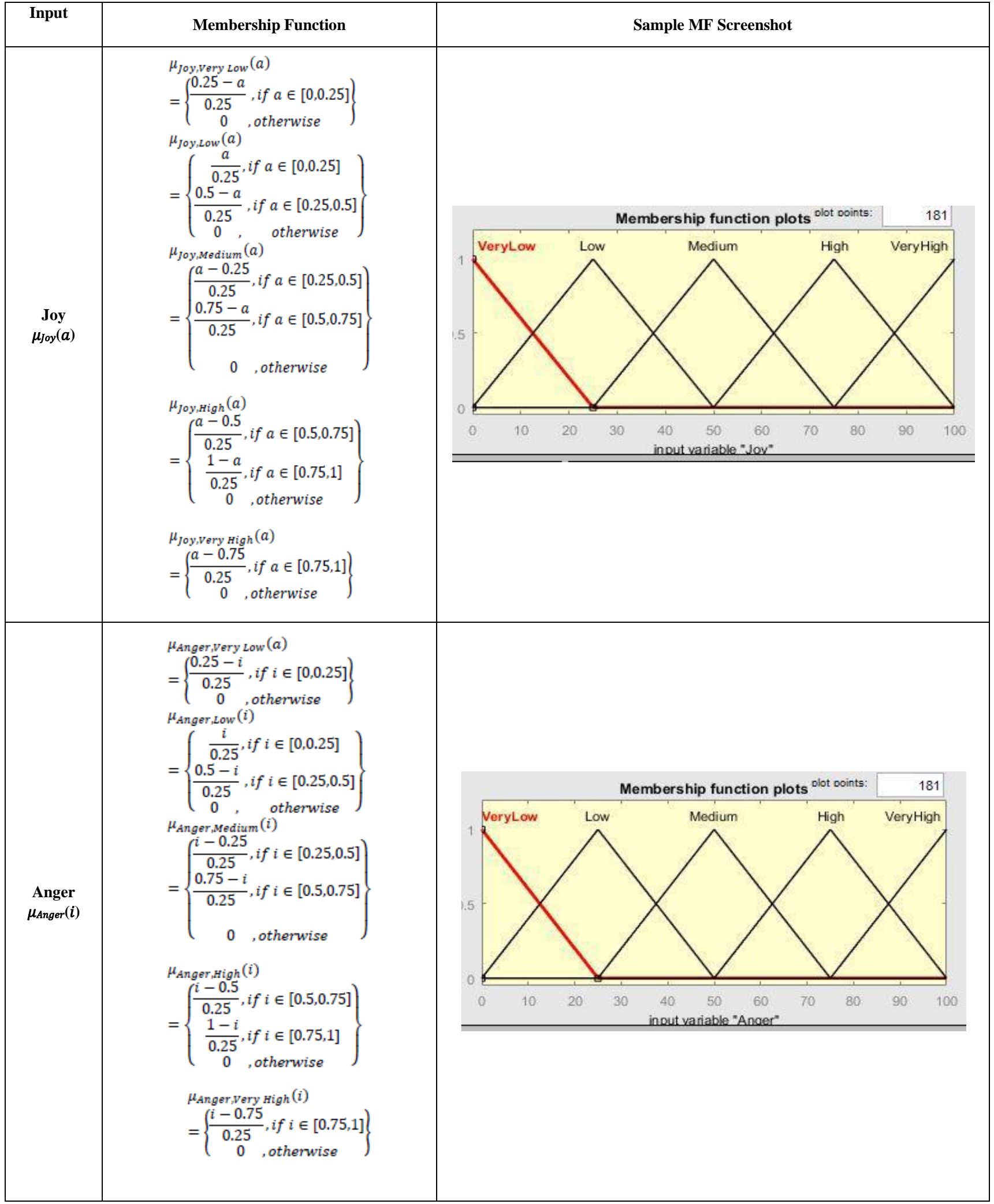

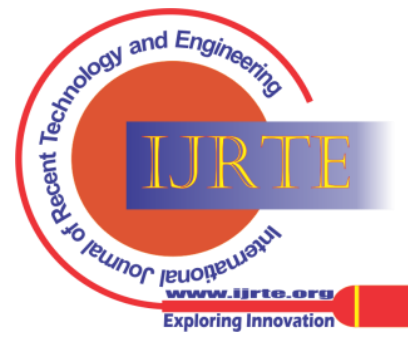




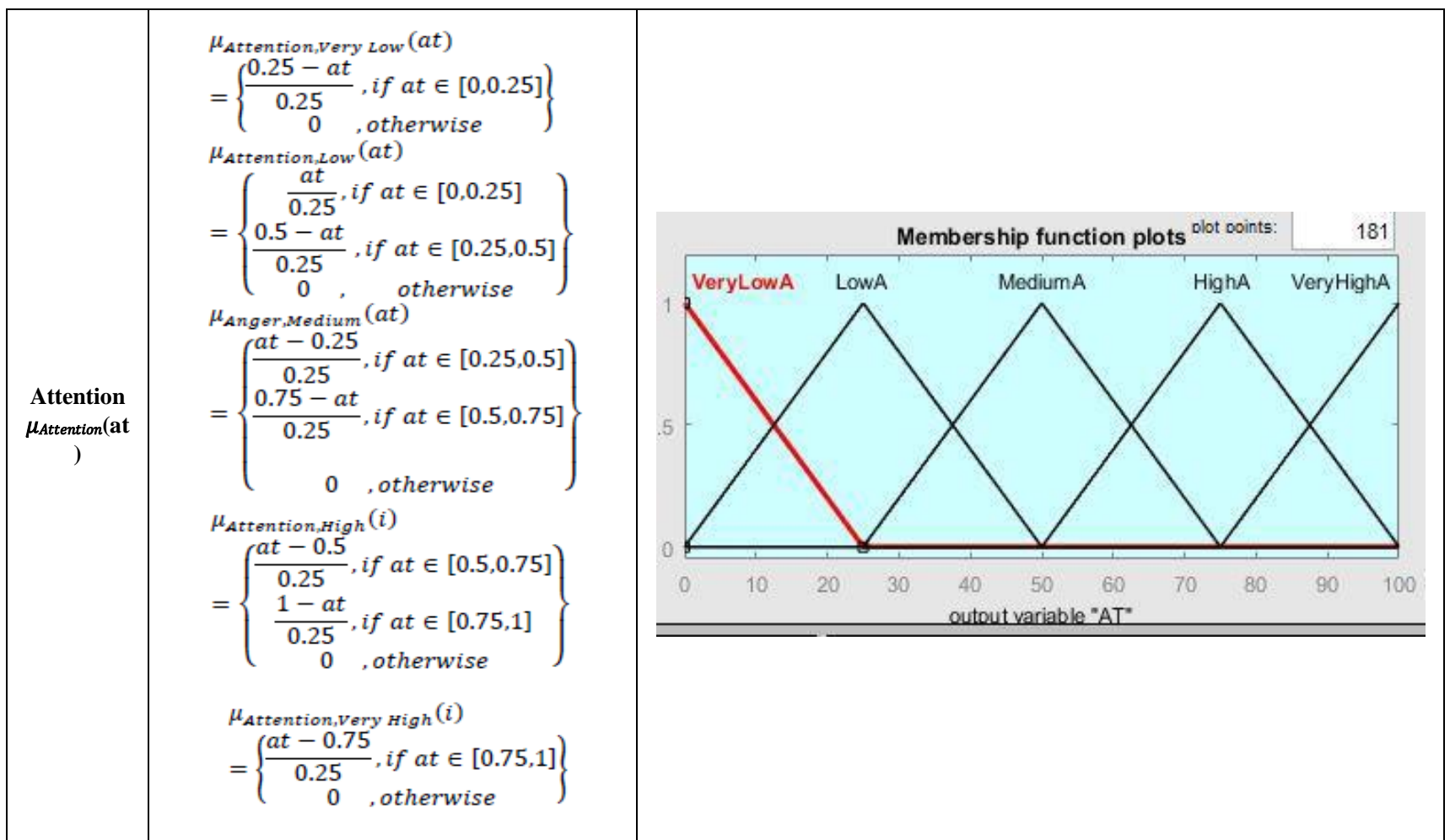

\subsection{Defuzzification}

To check the correctness of rules written in Mamdani fuzzy inference system for calculating attention, the process of defuzzification in contrast to fuzzification has been applied. After applying all mathematical equations and expressions the error rate we get is only $6 \%$, which shows the correctness of Mamdani fuzzy rules.

\section{EXPERIMENTATION AND RESULTS}

\subsection{Single Face Detection}

The testing data contains sets, each set having the face of a human. The face is detected from 34 points on a human face.

\subsection{Multiple Face Detection}

Detection of faces is not limited to only one face rather the application can detect more than two faces.

This shows the robustness in the application. The criteria for detecting face is that it can detect a face in live camera feed through 34 points on the face.

\subsection{Various Emotions On Face}

Once a face is detected, emotions on that face showing in the form of a graph. The various emotions of a person respectively change on the graph too. The emotion on the face is said to be joy, anger, disgust, etc. This categorization helps to define the attention.

\subsection{Attention To A Face}

Once multiple faces are detected through the live camera feed, the attention algorithm will decide to whom attention should be given. For Example, 3 faces detected through live camera feed, 2 faces have normal emotions but one face has a superb value of joy then the attention algorithm will make a red square around that face showing the sign of attention. As different persons have different levels of emotions, so this attention might be moved to another person if he/she will show extraordinary emotion.

\subsection{Attention On A Graph}

Every face has an attention rate according to the emotions on its face. The attention rate for a specific face is calculated using Fuzzy Logic as discussed in the above chapters. This attention rate is showing in the form of a graph. If there are two faces in the camera frame, each face has its graph associated with it. The graph is showing all emotions continuous values along with continuous attention vale for each face. As emotions are detecting through live camera feed, the attention values for making a graph are continuous.

\section{CONCLUSION}

The initial motive was to develop a complete system i.e. Hardware plus Software along with a machine learning algorithm. Also to create human-like attention based on emotions, as humans pay attention based on emotions, in robots. Based on machine learning algorithms, the robot can pay desired attention in real-time. After giving Attention the robot calculates the percentage of mouth opened and then feeds the disabled accordingly. The individual attention module has been completed. Both for single face and multiple faces, the attention criteria is the same. The attention module is working on a video (live camera feed) as input and gives an output graph showing values of emotions and attention for each face detected in the live camera feed. Calculating the percentage of opened mouth is the baseline of this project and all the information that can be derived from face has been covered like open eyes, brightness, head orientation and all emotions (joy, anger, sadness, disgust, surprise, and fear) are the salient features from face and its near environment. Attention graph is being maintained for every person in the video frame. 


\section{Future Work}

Make hardware flexible enough that the robot can adjust itself according to the height of the disabled.

The robot can be made to target the mouth of the disabled to feed him/her. Such motion can be introduced as the disabled falls forward, the robot moves backward, and vice versa. Addition of the mechanism to provide the ease of drinking water can be made. A robot can be made to handle variable distances while feeding.

\section{Acknowledgments}

This work is partially supported by the National Natural Science Foundation of China (No.61803251), the Educational Commission of Anhui Province of China(No.KJ2016A208), Anhui Province of China (KJ2016A200), Major Science and Technology Project of Anhui Province of China (16030901012), and key research and development project of Anhui Province of China (201904a05020092).

\section{REFERENCES}

1. Maja MATARIĆ, Adriana TAPUS, Carolee WEINSTEIN, Jon ERIKSSON, Socially Assistive Robotics for Stroke and Mild TB Rehabilitation, a Department of Computer Science, University of Southern California, USA b Division of Biokinesiology and Physical Therapy, University of Southern California, USA(2009)

2. Maja J Matarić, Jon Eriksson, David J Feil-Seifer, Carolee J Winstein, Socially assistive robotics for post-stroke rehabilitation, Computer Science Department, University of Southern California, Los Angeles, CA, USA and Department of Neurology, University of Southern California, Los Angeles, CA, USA(2007)

3. Daehyung Parka, Yuuna Hoshia, Harshal P. Mahajanb, Ho Keun Kima, Zackory Erickson, Wendy A. Rogers, Charles C. Kempa, Daehyung Parka, Yuuna Hoshia, Harshal P. Mahajanb, Ho Keun Kima, Zackory Erickson, Wendy A. Rogers, Charles C. Kempa, Daehyung Parka, Yuuna Hoshia, Harshal P. Mahajanb, Ho Keun Kima, Zackory Erickson, Wendy A. Rogersc, Charles C. Kempa(2019)

4. Won-Kyung Song and Jongbae Kim, Novel Assistive Robot for SelfFeeding, Korea National Rehabilitation Research Institute, Korea National Rehabilitation Center Korea(2012)

5. Derek McColl and Goldie Nejat, Meal-Time with a Socially Assistive Robot and Older Adults at a Long-term Care Facility, Meal-Time with a Socially Assistive Robot and Older Adults at a Long-term Care Facility(2013)

6. Homeyra Pourmohammadali, Jonathan Kofman, Amir Khajepour, DESIGN OF A MULTIPLE-USER INTELLIGENT FEEDING ROBOT FOR ELDERLY AND DISABLED PEOPLE, Dept. of Mechanical Eng. University of Waterloo Canada(2007)

7. Daehyung Park*, You Keun Kim, Zackory M. Erickson, and Charles C. Kemp, Towards Assistive Feeding with a General-Purpose Mobile Manipulator,(2016)

8. Tapomayukh Bhattacharjee, Maria E. Cabrera, Anat Caspi, Maya Cakmak, Siddhartha S. Srinivasa, A Community-Centered Design Framework for Robot-Assisted Feeding Systems, Paul G. Allen School of CSE, University of Washington, Seattle, USA(2019)

9. Alexandre Candeias, Travers Rhodes, Manuel Marques, Joao P. Costeira, Manuela Veloso, Vision Augmented Robot Feeding, ISR IST Universidade de Lisboa, Lisbon, Portugal, Carnegie Mellon University, Pittsburgh, USA(2018)

10. Matei Ciocarlie, Kaijen Hsiao, E. Gil Jones, Sachin Chitta, Radu Bogdan Rusu, and Ioan A. S, can, Towards Reliable Grasping and Manipulation in Household Environments, Rice University, Houston, TX, USA(2014)

11. Tiffany L. Chen, Matei Ciocarlie, Steve Cousins, Phillip Grice, Kelsey Hawkins, Kaijen Hsiao, Charles C. Kemp, Chih-Hung King, Daniel A. Lazewatsky, Adam Leeper, Hai Nguyen, Andreas Paepcke, Caroline Pantofaru, William D. Smart, and Leila Takayama, Robots for Humanity: Using Assistive Robots to Empower People with Disabilities(2013)

12. Phillip M. Grice, Andy Lee, Henry Evans, and Charles C. Kemp, The House: A Wearable Wince Detector to Stop Assistive Robots, 2012 IEEE RO-MAN: The 21st IEEE International Symposium on Robot and Human Interactive Communication. September 9-13, 2012. Paris, France. (2012)

13. Tapomayukh Bhattacharjee, Gilwoo Lee, Hanjun Song, and Siddhartha S. Srinivasa, Towards Robotic Feeding: Role of Haptics in Fork-based Food Manipulation, IEEE ROBOTICS, AND AUTOMATION LETTERS. (2019)

14. Filippo Arrichiello, Paolo Di Lillo, Daniele Di Vito, Gianluca Antonelli, Stefano Chiaverini, Assistive robot operated via P300based Brain Computer Interface, he Department of Electrical and Information Engineering of the University of Cassino and Southern Lazio, Via G. Di Biasio 43, 03043 Cassino (FR), Italy(2017)

15. Adam Leeper, Kaijen Hsiao, Matei Ciocarlie, Leila Takayama, Willow Garage, David Gossow, Strategies for Human-in-the-Loop Robotic Grasping(2012)

16. Phillip M. GriceID, Charles C. Kemp, In-home and remote use of robotic body surrogates by people with profound motor deficits, Department of Biomedical Engineering, Georgia Institute of Technology, Atlanta, GA, United States of America,( 2019)

17. Daehyung Park, Hokeun Kim, Yuuna Hoshi, Zackory Erickson, Arie Kapusta, and Charles C. Kemp, A Multimodal Execution Monitor with Anomaly Classification for Robot-Assisted Feeding, Georgia Institute of Technology, Atlanta, GA(2017)

18. Sebastian Schroer Ingo Killmann Barbara Frank Martin Volker Lukas Fiederer Tonio Ball Wolfram Burgard, An Autonomous Robotic Assistant for Drinking, e University of Freiburg, 79110 Freiburg, Germany(2016)

19. Jayakorn Vongkulbhisal, Fernando De la Torre, Joao P. Costeira, Discriminative Optimization: Theory and Applications to Point Cloud Registration, ISR - IST, Universidade de Lisboa, Lisboa, Portugal Carnegie Mellon University, Pittsburgh, PA, USA(2017)

20. Tapomayukh Bhattacharjee, Gilwoo Lee, Hanjun Song* , Siddhartha S. Srinivasa, Towards Robotic Feeding: Role of Haptics in Forkbased Food Manipulation, E ROBOTICS, AND AUTOMATION LETTERS. PREPRINT VERSION. (2019)

21. Peng Chang, Tas,kın Padır, Sim2Real2Sim: Bridging the Gap Between Simulation and Real-World in Flexible Object Manipulation, College of Engineering Northeastern University Boston, Massachusetts, USA(2020)

22. Jordi Pages, Luca Marchionni, Francesco Ferro, TIAGo: the modular robot that adapts to different research needs(2018)

23. Russell Toris, Julius Kammerl, David V. Lu, Jihoon Lee, Oddes Chadwick Jenkins, Sarah Osentoski, Mitchell Wills, and Sonia Chernova, Robot Web Tools: Efficient Messaging for Cloud Robotics(2015)

\section{AUTHOR PORFILE}

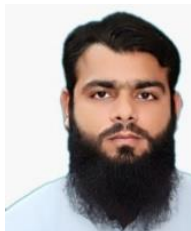

Mubashar Nawaz is a Master's student in Mechanical Engineering at Anhui university of science and technology, China. He completed his Bachelor's degree in Mechanical technology at The University of Lahore, Pakistan. His research area is Robot Design and Control, Robot kinematics, and Dynamics Robotics arm, Robotics Manipulator, assistive and industrial Robots.

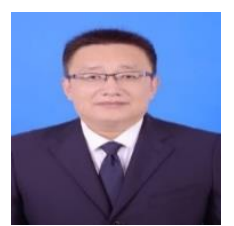

Xianhua Li is a Professor in Mechanical Engineering at the University of Science and Technology. He obtained a Ph.D. majoring in Mechatronics Engineering at Shanghai University in China. He taught Mechanical Engineering relevant subjects at the School of Mechanical Engineering and School of Artificial Intelligence. His research area is Robotics including Robot Design and Control, Robot Kinematics and Dynamics, Robot Arms Coordinated Operation and Control, Robot Tip-over, and Slip Avoidance.

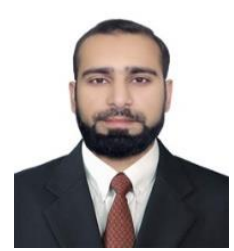

Sohaib Latif is a Doctorate student in Information Security Engineering at the Anhui University of Science and Technology. He has M. Phil. in Computer Science from The University of Lahore, Pakistan.

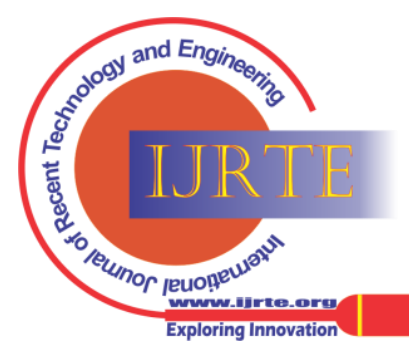


He taught Computer Science relevant subjects in the government and private sector of Pakistan for six years. His research interest includes Wireless Sensor Networks, MANET's, Petri net, Robotics, Deep Learning, and Internet of Things.

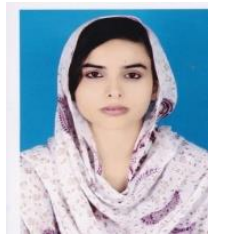

Sadaf Irshad is a Doctorate student in the School of Mathematics and Big Data at Anhui University of Science and Technology, China. She completed her Master's degree in Mathematics at the Institute of Southern Punjab, Multan, Pakistan. Her research area is Differential equations, complex designs, Robot Design, kinematics, and Dynamics.

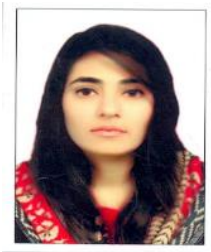

Shabnam Shahzadi is a Doctorate student in the School of Mathematics and Big Data at Anhui University of Science and Technology, China. She completed her Master's degree in Statistics at Arid Agriculture University, Rawalpindi, Pakistan. Her research areas are Stochastic Petri Nets, Data management, Advance Statistics, Complex Designs, Probability Theory, Statistical Inference.

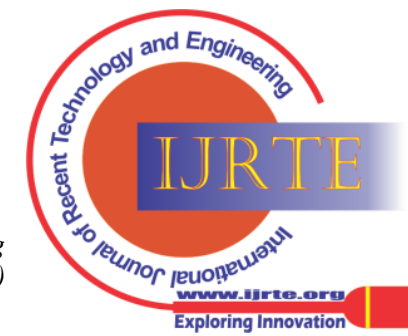

